Revue d'histoire de l'Amérique française

ZFS REVUE D.HISTOIRE DE L'AMÉRIQUE FRANÇAISE

\title{
Georges Pelletier et les questions économiques (1910-1929)
}

\section{Paul-André Linteau}

Volume 23, numéro 4, mars 1970

URI : https://id.erudit.org/iderudit/302935ar

DOI : https://doi.org/10.7202/302935ar

Aller au sommaire du numéro

Éditeur(s)

Institut d'histoire de l'Amérique française

ISSN

0035-2357 (imprimé)

1492-1383 (numérique)

Découvrir la revue

Citer cet article

Linteau, P.-A. (1970). Georges Pelletier et les questions économiques (1910-1929). Revue d'histoire de l'Amérique française, 23(4), 583-600. https://doi.org/10.7202/302935ar d'utilisation que vous pouvez consulter en ligne.

https://apropos.erudit.org/fr/usagers/politique-dutilisation/ 


\section{GEORGES PELLETIER ET LES QUESTIONS ÉCONOMIQUES (1910-1929)*}

Les études consacrées à la pensée de l'élite nationaliste traditionnelle du Québec sont encore peu nombreuses. Jusqu'ici on a abordé cette question dans une perspective assez globale. Il faut maintenant, dans une seconde étape, vérifier par une analyse plus poussée les hypothèses émises, les interprétations avancées par les historiens ou les critiques de cette élite. ${ }^{1}$ L'étude de la pensée de Georges Pelletier est une contribution, parmi d'autres ${ }^{2}$, à cette analyse en profondeur de la pensée de l'élite nationaliste traditionnelle.

Le nom de Georges Pelletier est intimement lié à celui du journal Le Devoir où il a occupé successivement les postes de correspondant parlementaire à Ottawa (1910), secrétaire de la

* Ce texte résume les conclusions d'une thèse de maîtrise présentée en 1969. J'ai pu consulter le fonds Georges Pelletier et le fonds Lionel Groulx, déposés à l'Institut d'histoire de l'Amérique française. Je remercie Madame Juliette Rémillard et Mademoiselle Madeleine Dionne du secrétariat de l'Institut de leur collaboration. Je remercie également Monsieur Maurice Séguin qui a guidé cette recherche, Monsieur René Durocher qui a accepté de lire et de commenter le texte de cet article et Monsieur Paul Sauriol qui m'a fait d'utiles remarques.

1 Parmi ces études signalons: Pierre Elliott Trudeau, "La province de Québec au moment de la grève", La grève de l'amiante (Montréal, Cité libre, 1956), 1-91; André Laurendeau, "Sur cent pages de Pierre Elliott Trudeau", Le Devoir, 6 octobre, 10 octobre, 11 octobre 1956; Michel Brunet, "Trois dominantes de la pensée canadienne-française: l'agriculturisme, l'anti-étatisme et le messianisme", La présence anglaise et les Canadiens (Montréal, Beauchemin, 1964), 113-166; François-Albert Angers, dir., "50 années de nationalisme positif", numéro spécial de l'Action nationale, LII, 7-8 (mars-avril 1963) : 641-903.

2 Paul-André Linteau, La pensée économique et sociale de Georges Pelletier, 1910-1929. Thèse de M.A., Université de Montréal, 1969, 197 p.; Pierre Dandurand, Analyse de l'idéologie d'un journal nationaliste canadien-français: Le Devoir, 1911-1956. Thèse de M.A., Université de Montréal, 1961, 186 p.; Yves Saint-Germain, La prospérité nord-américaine de l'aprèsguerre. Le Québec et les Canadiens français (1919-1927). Thèse de M.A., Université de Montréal, 1963, 148 p.; Marie-Lise Brunel-Guitton, La pensée historique de Lionel Groulx. Thèse de M.A., Université de Montréal, $1969,195 \mathrm{p}$. Signalons aussi quelques thèses en préparation : celle de Susan Robertson sur l'Action française, celle de Peter Southam sur Olivar Asselin et celle de Laurier Renaud sur L'ACJC.

Vol. xxIII, no 4 (mars 1970) 
rédaction (1915), administrateur-gérant (1924) et directeur (1932). La pensée de Pelletier est d'autant plus intéressante à étudier qu'il a pu l'exprimer quotidiennement, pendant plus de trente ans, dans un organe dont l'influence a été considérable, bien que difficile à évaluer.

L'attitude de notre élite nationaliste face aux questions économiques a fait l'objet d'intéressantes controverses. A titre de journaliste, Pelletier s'est penché sur les problèmes économiques. L'examen de ses commentaires nous permettra de cerner un peu mieux les convictions d'un représentant de ce groupe. Nous avons limité notre étude à la période 1910-1929, soit des débuts de Pelletier au Devoir jusqu'à la crise économique. Cette dernière marque en effet une étape importante dans la pensée de l'élite traditionnelle et elle mérite qu'on lui consacre une étude particulière.

Il ne faudrait pas passer sous silence le fait qu'une très grande partie des articles de Pelletier ont été consacrés à des questions politiques. On se rappellera ses chroniques parlementaires d'Ottawa, ses portraits politiques, ses commentaires sur les questions internationales et ses nombreux autres écrits abordant des questions politiques. Sans nier l'intérêt d'étudier cet aspect de la pensée de Pelletier, la présente recherche se limite aux thèmes économiques que Pelletier a développés dans ses écrits.

Quelques problèmes d'ordre économique attirent l'attention de Pelletier mais souvent il s'attarde plutôt à leurs conséquences sociales. L'immigration et le coût de la vie le préoccupent beaucoup au cours des années qui précèdent immédiatement la Grande Guerre. Le journaliste s'intéresse également, à diverses époques, à plusieurs aspects de l'économie canadienne: canaux, chemins de fer, banques, économie de guerre, dette publique et tarif. Enfin, au cours des années d'après-guerre, il aborde le problème de la situation économique des Canadiens français, de leur rôle dans l'industrie et l'agriculture.

\section{L'immigration}

$\mathrm{Au}$ cours de la décennie précédant la première guerre mondiale, le Canada accueille une vague d'immigration qui dépasse en ampleur tout ce que le pays a connu auparavant et qui atteint un sommet en 1913, année où on constate l'arrivée de plus de 400,000 immigrants. Cette situation crée de sérieux problèmes 
d'intégration. En 1911, la population née à l'étranger représente 22 pour cent de la population canadienne totale. ${ }^{3}$ Cette immigration a de plus en plus un caractère hétérogène et plus de la moitié des nouveaux venus se dirigent vers les provinces de l'Ouest.

Les Nationalistes ne tarderont pas à souligner les problèmes que soulève ce mouvement migratoire. A la Chambre des Communes, Bourassa et Lavergne attaquent avec vigueur la politique d'immigration du gouvernement fédéral. Leurs reproches portent sur le trop grand nombre d'immigrants, la composition ethnique trop hétérogène des nouveaux venus, la mauvaise qualité de l'immigration britannique et enfin l'absence d'efforts en vue de stimuler la venue d'immigrants français."

Dans Le Devoir, Georges Pelletier s'intéressera de près à cette question. En 1913, après avoir fait enquête, il publie une série de vingt articles qui seront ensuite édités en brochure. ${ }^{5}$ Pelletier ne s'oppose pas à l'immigration. Il considère que "la vaste superficie du Canada exige une main-d'œuvre de plus en plus grande, pour développer ses ressources naturelles, ouvrir de nouveaux territoires et accélérer la production des fermes et des métairies" ${ }^{6}$. Mais, selon lui, cette immigration devient dangereuse quand elle est trop forte. Elle entraîne alors une perte d'influence des populations initiales et compromet le délicat équilibre politique établi au Canada au cours du siècle précédent. Il constate la difficulté d'intégrer ces nouveaux venus à cause de leur forte proportion par rapport à la population originale et à la suite de leur établissement dans les régions vides de l'Ouest. ${ }^{7}$ Sa solution sera de restreindre le nombre d'immigrants en pratiquant une sélection rigoureuse.

Quels seront ses critères de choix ? Pelletier considère comme indésirables non seulement les catégories de gens que la loi rejette déjà (malades mentaux, prostituées, mendiants, etc.), mais également l'immigrant "que la nation ne pourra jamais assimiler, à cause de ses origines, de ses mœurs, de sa civili-

3 M. C. Urquhart et K. A. H. Buckley, Historical Statistics of Canada (Toronto, Macmillan, 1965), 19.

4 Débats des Communes 1906, II : 2501-2508; 1906-07, IV : 6329-6340, 6355-6372.

5 Georges Pelletier, L'immigration canadienne (Montréal, Le Devoir, 1913), 73 p. A l'avenir: L'immigration.

${ }^{6}$ Le Devoir, 2 octobre 1912, 1.

7 L'Immigration, 60. 
sation différente de la nôtre" 8 . Dans ce dernier groupe qu'il faudrait, selon lui, "impitoyablement rejeter" parce qu'inassimilable, Pelletier classe, entre autres, les Noirs, la plupart des Asiatiques et des Juifs, les Siciliens. L'immigrant par excellence sera l'agriculteur, venant de préférence des régions du nord de l'Europe. ${ }^{9}$

Dans ses divers articles Pelletier s'attaque surtout à la politique du gouvernement fédéral à qui il reproche de ne pas exercer un contrôle assez rigoureux sur les immigrants. Il propose une série de réformes : un examen médical beaucoup plus sévère, une enquête sur la valeur morale des immigrants. Pelletier a tendance à établir un lien direct entre la vague d'immigration et la hausse de la criminalité. Il espère remédier à la criminalité en exigeant de l'immigrant un casier judiciaire vierge ou un certificat de moralité. ${ }^{10}$ Pelletier a tendance à confondre les conséquences sociales de l'industrialisation et de l'urbanisation avec celles de l'immigration. Dans l'ensemble, les critiques que le journaliste du Devoir adresse au gouvernement portent sur des questions de détail.

Pelletier s'intéresse donc beaucoup plus aux conséquences sociales de l'immigration qu'à son apport économique. Il reconnaît bien sûr la contribution des immigrants au développement économique, mais il ne s'étend guère sur ce sujet. En 1913 il voit dans la politique d'immigration du gouvernement fédéral l'une des causes les plus importantes de la hausse du coût de la vie. ${ }^{11}$ Plus tard, il adopte une position inverse en souhaitant l'abaissement des salaires grâce au maintien au Canada d'une masse d'ouvriers étrangers qui exerceraient une pression à la baisse sur le coût de la vie. ${ }^{12}$ En période de difficulté économique, comme c'est le cas en 1920-22, Pelletier ne désire pas stimuler l'immigration. "Nous ne sommes pas prêts à recevoir un nouvel afflux d'immigrés, nous ne saurons où les loger, comment les placer, comment les faire travailler." ${ }^{13}$ Pendant toute la décennie d'après-guerre, il insiste pour que le Canada attire des immigrants agricoles plutôt qu'urbains. Au début de

8 Ibid., 7.

9 Ibid., 5-7.

${ }^{10}$ Le Devoir, 20 janvier 1910, 2.

11 Ibid., 19 mars 1913, 1.

12 Ibid., 10 mars 1919, 1.

13 Ibid., 21 mars 1921, 1. 
1929, il déclare encore que les immigrants ruraux sont les meilleurs. ${ }^{14}$

L'optique dans laquelle il aborde l'immigration est liée à un certain idéal de société: une société vivant de la vie simple et rude des champs, où les individus tarés (socialement, physiquement, mentalement) seraient presque inexistants, où le pluralisme a un droit de cité assez restreint. Pelletier s'est donc attaqué aux aspects de l'immigration qui pouvaient compromettre cet idéal de société. Ce faisant, il paraît avoir gaspillé ses énergies sur des questions assez secondaires. Les criminels, les aliénés, les souteneurs, les prostituées ne représentaient qu'une faible partie des millions d'immigrants venus au Canada. En concentrant son attention sur des sujets aussi limités, il a négligé des problèmes d'une autre ampleur comme ceux de l'intégration économique et sociale de l'immigrant. Il a plus insisté sur les effets négatifs que positifs de l'immigration, bien que ses critiques soient plus nuancées que celles de Lavergne ou de Bourassa. Il ne fait qu'effleurer certaines des questions soulevées par les orateurs nationalistes, par exemple l'immigration française, la colonisation de l'Ouest par des Canadiens, la mauvaise qualité de l'immigration britannique.

\section{Le coût de la vie}

Si Pelletier aborde rapidement les aspects économiques du problème de l'immigration, il n'en est pas de même dans le cas du coût de la vie. Pendant le premier conflit mondial et au cours des années qui le précèdent, le coût de la vie connaît une hausse sensible au Canada. En 1913, le gouvernement fédéral crée une commission d'enquête pour étudier les problèmes causés par cette hausse. La commission remettra son rapport en $1915 .{ }^{15}$ Pelletier s'intéresse à cette question d'actualité et publie dans Le Devoir, du 9 au 31 décembre 1913, une série de quinze articles sous le titre "Pourquoi la vie est chère". Il y ajoutera les résultats d'une enquête sur le prix du pain en 1916. Enfin il publie une brochure sur le coût de la vie en $1917 . .^{16}$ Il consacre aussi de nombreux autres articles à cette question.

En 1913, il s'interroge sur les causes de la hausse du coût de la vie. Il croit d'ailleurs que c'est beaucoup plus l'ensemble

14 Ibid., 13 mars 1929, 1.

15 Canada, Board of Inquiry into Cost of Living, Report of the Board (2 vol., Ottawa, 1915).

16 Georges Pelletier. Tout est cher... Pourquoi? (1910-191\%) (Montréal, Le Devoir, 1917), 60 p. 
des causes, qu'une seule d'entre elles, qui explique la situation. Plusieurs motifs invoqués habituellement pour expliquer la hausse de l'indice des prix ne semblent guère le satisfaire. Il n'accorde qu'une importance marginale à des facteurs comme le tarif ${ }^{17}$, l'action des trusts ${ }^{18}$, l'apparition des entrepôts frigorifiques ${ }^{19}$, les taux de transport ${ }^{20}$ ou la dépréciation de l'or ${ }^{21}$. Il considère comme beaucoup plus déterminantes l'augmentation des dépenses gouvernementales et la politique d'immigration du gouvernement fédéral. ${ }^{22}$ Il insiste surtout sur l'accroissement de la population urbaine qui "est improciuctive, au point de vue de la richesse alimentaire de la nation" ${ }^{23}$. La société normale, selon le journaliste du Devoir, doit être marquée par un équilibre "entre la population des villes et celle des campagnes, entre la production et la consommation, si l'on ne veut pas que le prix des denrées s'accroisse outre mesure" ${ }^{24}$. Cet équilibre, dont la nature reste assez nébuleuse, lui apparaît maintenant rompu, ce qui entraîne une hausse du coût de la vie. Il ne mentionne pas, en 1913, qu'une baisse de la population agricole puisse être compensée par une augmentation de la productivité. ${ }^{25}$ Parmi les autres facteurs qui peuvent expliquer la hausse du coût de la vie, Pelletier signale l'existence des nombreux intermédiaires, ${ }^{26}$ les changements d'habitudes de la population dont il stigmatise "le gaspillage et les extravagances" ${ }^{27}$, l'influence de la spéculation immobilière ${ }^{28}$ et enfin l'insuffisance de la production agricole. ${ }^{29}$ Pelletier termine son enquête de 1913 en traçant un programme d'action dans lequel il énumère les principales tâches de chacun. Aux individus, il prêche l'économie et propose la formation de coopératives. Les devoirs qu'il trace aux municipalités se résument ainsi: favoriser le développement de marchés publics, inspecter les entrepôts frigorifiques, décourager la spéculation

${ }^{17}$ Le Devoir, 10 décembre 1913, 1.

18 Ibid., 12 décembre 1913, 1-2.

19 Ibid., 15 décembre 1913, 1-2.

20 Ibid., 16 décembre 1913, 1-2.

21 Ibid., 17 décembre 1913, 1-2.

22 Ibid., 19 décembre 1913, 1-2.

23 Ibid., 20 décembre 1913, 1.

24 Ibid., 22 décembre 1913, 1.

25 Il soulèvera cet argument en 1922 et tentera d'en minimiser l'importance. Le Devoir, 5 juillet 1922, 1.

26 Ibid., 23 décembre 1913, 1.

27 Ibid., 26 décembre 1913, 1.

28 Ibid., 27 décembre 1913, 1-2.

29 Ibid., 29 décembre 1913, 1-2. 
immobilière et construire des logements salubres pour les ouvriers. Les gouvernements provinciaux, de leur côté, doivent encourager l'agriculture par l'enseignement agricole et l'aide à la colonisation, favoriser le développement des coopératives et organiser le crédit rural. Quant au gouvernement fédéral il devrait réduire le tarif, contrôler plus étroitement les trusts, limiter les dépenses publiques, restreindre l'immigration et collaborer avec les provinces dans le domaine agricole. Pelletier réclame une action immédiate pour enrayer la hausse du coût de la vie. ${ }^{30}$

En abordant un sujet aussi complexe, à une époque où les économistes sont presque inexistants au Québec, Pelletier fait preuve de vigueur intellectuelle et d'un intérêt certain pour les questions économiques. Il fait un effort sérieux pour comprendre les problèmes économiques, mais son étude comporte de sérieuses lacunes qui proviennent d'une évaluation incomplète de la réalité économique. En 1913, Pelletier n'a pas saisi dans toute son ampleur la signification des transformations économiques des deux décennies précédentes. Il n'a pas compris à quel point elles ont modifié, et de façon permanente, la société. Le journaliste rêve d'autarcie, il demande le contact direct entre le producteur et le consommateur, il désire un équilibre rural-urbain où le premier serait encore le plus important. Ce faisant, il ne saisit pas ces traits de l'économie moderne que sont la spécialisation des tâches et des régions, l'amélioration des techniques et de la productivité, l'urbanisation qui se fait de façon inexorable. En centrant son attention sur la production agricole, il néglige d'autres secteurs productifs dont l'effet d'entraînement est important.

Pelletier n'est pas le seul responsable des faiblesses de son analyse. Il exprime des idées de son temps, qu'il emprunte à divers auteurs. N'étant pas économiste, il ne peut critiquer de façon rigoureuse ses sources d'information. D'ailleurs, la commission d'enquête créée par le gouvernement fédéral et qui remettra son rapport en 1915 aura des conclusions très proches de celles de Pelletier. Parmi les principales causes de la hausse du coût de la vie, elle placera la réduction du nombre des producteurs agricoles et son corollaire, l'urbanisation, ainsi que les dépenses extravagantes, le gaspillage, les dépenses de travaux publics et l'augmentation du stock d'or. ${ }^{31}$ Dans son histoire de

30 Ibid., 31 décembre 1913, 2.

31 Canada, Board of Inquiry into Cost of Living, Report of the Board. 
la pensée économique au Canada, C.D.W. Goodwin a jugé sévèrement les méthodes de travail de la commission. ${ }^{32}$ Si une commission d'enquête mise sur pied par l'Etat fédéral ne donne pas de résultats très brillants, il ne faut pas s'attendre à ce que Pelletier, simple journaliste et disposant de faibles moyens financiers, fasse beaucoup mieux. Le travail de Pelletier présente donc des faiblesses sérieuses, mais qui peuvent s'expliquer dans un contexte où la pensée économique au Canada, et au Québec, était encore peu développée. ${ }^{33}$

Un autre aspect intéressant de l'action intellectuelle de Pelletier est sa vigoureuse défense des droits du consommateur. Ses études sur le coût de la vie l'amènent à constater que c'est le consommateur et surtout le gagne-petit qui en portent tout le poids. Il signale, dans Le Devoir, les domaines où le consommateur est victime d'exploiteurs. Il s'en prend surtout aux industriels et aux spéculateurs qui profitent de la situation extraordinaire, en temps de guerre, pour faire des profits exorbitants sur les denrées alimentaires: "celui qui spécule sur la faim d'autrui est l'un des plus vilains personnages qui soient" 34 . C'est ainsi qu'il s'oppose inlassablement et avec vigueur aux augmentations des prix du pain. En plus de nombreux blocsnotes, il consacre à cette question une série de dix articles publiés sous le titre "Notre pain quotidien". 35

Pour améliorer la situation du consommateur, dans son ensemble, Pelletier insiste sur les solutions individuelles: restriction des dépenses de luxe ${ }^{36}$, pratique de l'économie ${ }^{37}$, retour à la terre, pour les familles nombreuses ${ }^{38}$. Il est aussi conscient du rôle que peuvent jouer les initiatives collectives: coopératives ${ }^{39}$ et ligues de consommateurs ${ }^{40}$. Quant à l'Etat, il lui assigne surtout un rôle de police et de réglementation. ${ }^{41}$ Ainsi

32 C.D.W. Goodwin, Canadian Economic Thought. The Political Economy of a Developing Nation, 1814-1914 (Durham, Duke University Press, 1961), 140.

33 Ibid., passim.

34 Le Devoir, 25 octobre 1915, 1.

35 Ibid., 21 août au 31 août 1916.

36 Ibid., 16 juin 1914, 1.

37 Ibid., 28 août $1914,1$.

38 Georges Pelletier, "Le budget familial", Semaines sociales du Canada, IVe session, 1932: 99-100.

39 Le Devoir, 27 janvier 1917, 1.

40 Ibid., 30 septembre 1915, 1.

41 Ibid., 25 octobre 1915, 1; 12 mars 1918, 1. 
Georges Pelletier cherche à améliorer le sort du consommateur et surtout du petit salarié, victime sans défense des fluctuations économiques.

\section{L'économie canadienne}

Outre ses grandes enquêtes sur l'immigration et le coût de la vie, Georges Pelletier a consacré plusieurs textes, éditoriaux ou blocs-notes, à divers problèmes de l'économie canadienne. C'est ainsi qu'il s'intéresse de près à des éléments de l'infrastructure économique du pays comme les canaux et surtout les chemins de fer. Il appuie avec vigueur le projet de canal de la baie Georgienne, projet qui jouit d'une certaine vogue au cours des années d'avant-guerre. ${ }^{42}$

Beaucoup plus importante est la question ferroviaire. De 1900 à 1915 le Canada connaît l'une de ses grandes périodes de construction ferroviaire et voit surgir deux nouveaux transcontinentaux. Pelletier ne manque pas de reprocher aux gouvernements l'imprévoyance et l'imprudence dont ils ont fait preuve dans leur politique de chemins de fer.

Nous sommes allés trop vite en besogne. L'optimisme exagéré de nos gouvernants croule, devant l'expérience coûteuse que nous leur avons laissé faire, en favorisant la construction, presque de toutes pièces, de deux transcontinentaux nouveaux dans des zones où le développement graduel et raisonné de celui qui existait déjà eût suffi. ${ }^{43}$

Il insiste encore sur cette imprévoyance dans plusieurs de ses articles au Devoir. Pendant la guerre, les difficultés financières du Canadian Northern et du Grand Trunk Pacific amènent le gouvernement fédéral à intervenir. $\mathrm{Au}$ cours de la période, des individus et des groupes réclament l'étatisation des chemins de fer. Pelletier se montre réticent, vu le bilan peu reluisant des chemins de fer gouvernementaux depuis la Confédération; il évite néanmoins de se prononcer sur le sujet.44 Le journaliste n'éprouve cependant aucune sympathie pour les deux compagnies en mauvaise posture financière. Il s'oppose au versement d'une indemnité pour l'achat des actions du Canadian Northern. ${ }^{45}$ En 1921, il paraît satisfait de la décision des arbitres qui ont déclaré sans valeur les actions du Grand Tronc. ${ }^{46}$

\footnotetext{
42 Ibid., 24 avril 1913, 1; 2 octobre 1913, 1; 12 janvier 1914, 1.

43 Ibid., 19 octobre 1915, 1.

44 Ibid., 21 septembre 1916, 1; 25 janvier 1918, 1.

45 Ibid., 24 août $1917,1$.

46 Ibid., 9 septembre 1921, 1.
} 
Critique acerbe de la politique ferroviaire, Georges Pelletier ne l'est pas moins quand il aborde le problème de la concentration bancaire. La réduction progressive du nombre des banques canadiennes ${ }^{47}$ lui fait peur et il craint la concentration des capitaux qui en résulte. Il propose plutôt la mise sur pied de banques régionales.

Des banques régionales ne vaudraient-elles pas mieux pour le public que de vastes banques concentrées dans deux ou trois provinces seulement du Canada, comme ce sera bientôt la situation? Croit-on que l'existence de grands comptoirs d'escompte superposés à des banques de district et à des banques régionales et leur réescomptant, le cas échéant, les effets de commerce qu'elles leur apporteraient n'assurerait pas plus de souplesse à notre régime économique et financier ? ${ }^{48}$

Le système des succursales, dit-il, ne favorise pas l'économie régionale puisque les décisions sont prises à Toronto et à Montréal. Le système des banques américaines lui apparaît mieux adapté aux besoins régionaux. ${ }^{4}$ Selon lui, les deux banques canadiennes-françaises représentent bien cet idéal de banques moyennes régionales dont il souhaite la formation au Canada.50

Les problèmes que soulève l'économie de guerre vont également retenir l'attention de Pelletier. Il attaque avec vigueur les profiteurs de guerre qui se taillent de jolies fortunes aux dépens des contribuables. Les profits des meuneries soulèvent l'ire du journaliste à une époque où le prix du pain est élevé. ${ }^{51}$ Il suggère l'imposition d'une taxe sur les profits réalisés grâce aux commandes de guerre. ${ }^{52} \mathrm{Il}$ demande au gouvernement de limiter les profits des fournisseurs de guerre à 10 ou 15 pour cent et de confisquer le surplus. ${ }^{53}$

Pendant la guerre, le Canada fournit des denrées alimentaires en grande quantité aux belligérants. Cette situation inquiète le journaliste du Devoir. Il craint qu'en pensant trop à ceux des Alliés le Canada ne puisse répondre à ses propres

47 On en compte 35 en 1901, 28 en 1911, 18 en 1921 et 10 en 1931.

M.C. Urquhart et K.A.H. Buckley, Historical Statistics of Canada, 246.

48 Le Devoir, 25 juillet 1928, 1.

49 Ibid.

50 Ibid., 2 août 1928, 1.

51 Ibid., 28 mai 1918, 1; 4 octobre 1918, 1; 23 octobre 1918, 1.

52 Ibid., 3 novembre 1915, 1.

53 Ibid., 14 février 1917, 1. 
besoins. Il suggère l'imposition d'un embargo sur l'exportation des produits alimentaires afin d'assurer au Canada les réserves nécessaires à son alimentation. ${ }^{54}$

Georges Pelletier songe également à préparer l'après-guerre. Il presse le Canada de "prendre avantage des circonstances actuelles pour développer considérablement ... sa production économique, agricole et industrielle" ${ }^{55}$. Prévoyant une crise économique à la fin du conflit il considère que deux "excellents moyens" pour en amoindrir l'impact sont "l'agriculture et la fondation de nouvelles industries, - pacifiques, celles-là, -". ${ }^{56}$

Mais la grande préoccupation de Pelletier, pendant la guerre, est l'augmentation rapide de la dette du Canada. Cette situation se poursuivra d'ailleurs bien après l'armistice. Dans plusieurs de ses articles au Devoir, il souligne que la dette est trop considérable et impose un lourd fardeau financier, que peu de gens s'inquiètent des conséquences de cet endettement, mais que les lendemains seront ardus avec les échéances auxquelles il faudra faire face. Selon Pelletier, la prospérité n'est qu'apparente. On ne peut parler de prospérité, dit-il, quand on s'endette de plus en plus. ${ }^{57}$ L'idée de faire payer par les générations futures les dépenses d'aujourd'hui ne lui sourit guère. ${ }^{58}$

L'augmentation des dépenses, pendant la guerre, amène le gouvernement canadien à créer, en 1917, l'impôt sur le revenu. Pelletier est entièrement d'accord avec le principe de ce type de taxation. Il réclame cependant que dans ses modalités la mesure frappe lourdement les riches et qu'elle épargne les petits salariés. ${ }^{59}$

Un dernier aspect de l'économie canadienne attire l'attention de Pelletier, la politique tarifaire. En fait, le journaliste hésite à se prononcer sur ce sujet épineux. Chaque fois que la question du tarif est débattue en Chambre ou sur la place publique, il expose les thèses en présence, sans prendre position de façon catégorique. Son attitude lors de l'enquête sur le coût de la vie ${ }^{60}$

54 Ibid., 27 février 1917, 1.

55 Ibid., 5 novembre 1915, 1.

56 Ibid., 8 juin 1916, 1.

57 Ibid., 11 septembre 1916, 1; 2 octobre 1916, 1.

58 Ibid., 7 août 1916, 1.

59 Ibid., 19 juillet 1917, 1.

60 Ibid., 10 décembre 1913, 1. 
permet de le classer comme protectionniste modéré, partisan du statu quo. En 1924, il demande que le tarif protège surtout les industries utilisant des matières premières canadiennes. "En résumé, ce sont les industries tout à fait de chez nous et tout à fait nationales qu'il faut défendre d'abord contre une concurrence étrangère faite à la faveur du dumping. Celles des lainages et des cuirs sont aux premiers rangs de celles-là." ${ }^{1}$

L'intérêt de Georges Pelletier se porte donc vers un grand nombre de questions qui touchent l'économie canadienne. Il la critique sévèrement, car la situation du Canada ne lui apparaît pas très brillante. Il s'attache à souligner l'imprévoyance du gouvernement qui a entrepris une coûteuse politique ferroviaire et qui a accru considérablement la dette publique. Il ne parle guère cependant des effets bénéfiques des politiques gouvernementales. Il se pose en défenseur du contribuable et du petit salarié en réclamant pour eux plus de justice.

\section{Agriculture et industrie : La situation économique des Canadiens français}

$\mathrm{Au}$ cours des premières décennies du $20^{\circ}$ siècle, le Québec prend de plus en plus le visage d'une société industrielle et urbaine. De nouveaux secteurs - l'hydroélectricité, les pâtes et papiers - se développent. La production manufacturière progresse nettement. La prise de conscience de ce phénomène d'industrialisation suscite une double réaction chez les penseurs canadiens-français. Certains sentent d'abord le besoin de confirmer la vocation agricole du Québec. Ils condamnent de façon non équivoque la "désertion des campagnes"; ils chantent les vertus de la vie rurale et de l'agriculture; ils proposent des réformes afin d'améliorer le sort de l'agriculteur. Cet état d'esprit, qualifié d'agriculturisme ${ }^{62}$, se manifeste par un foisonnement de publications. Les textes du père Alexandre Dugré sont très révélateurs de cet état d'esprit. ${ }^{63}$ D'autres penseurs canadiens-français acceptent cependant la nécessité d'une certaine industrialisation et veulent que leurs compatriotes n'y participent pas qu'en subalternes. On cite souvent à cet égard

61 Ibid., 5 mai 1924, 1.

62 Michel Brunet, "Trois dominantes de la pensée canadienne-française : l'agriculturisme, l'anti-étatisme et le messianisme", La présence anglaise et les Canadiens (Montréal, Beauchemin, 1964), 113-166.

63 Voir A. Dugré, Les avantages de l'agriculture (Montráal, L'Ecole sociale populaire, 1916). 
le plaidoyer fait en 1922 par Joseph Versailles. ${ }^{64}$ Deux réponses sont donc proposées: le retour à la terre et le désir de contrôler l'industrie pour la mettre au service de la population du Québec. Il n'y a cependant pas toujours une opposition très nette entre ces deux courants de pensée. Un grand nombre de penseurs ont tenté d'élaborer une position de compromis entre ces deux solutions. Malgré cet effort, plusieurs d'entre eux ont exagéré le rôle de l'agriculture et ont eu une vision limitée de celui de l'industrie.

Pelletier se prononce à plusieurs reprises sur l'équilibre qui doit exister dans une société - et pas seulement celle du Québec - entre la population urbaine et la population rurale. Un des fondements de sa pensée sur ce sujet est l'idée que l'agriculture a la primauté dans la vie économique d'une nation. Il déclare "que l'agriculture est de sa nature même l'industrie fondamentale d'un pays bien organisé" 65. Cette primauté de l'agriculture est surtout importante au Québec. Georges Pelletier croit "que la race canadienne-française est essentiellement rurale et qu'elle ne se développe comme elle le doit qu'en autant qu'elle vit à la campagne" 66 .

Le journaliste est frappé par le développement rapide des grandes villes, surtout Montréal et Toronto. Il tient en suspicion les citadins; ils ne font pas d'aussi bons citoyens que les ruraux. "La population des villes, formée en grande partie de déracinés, ne constitue pas un élément aussi sain, aussi posé, aussi réfractaire aux idées fausses et aux théories subversives, en règle générale, que les groupements ruraux." 67 Il en vient à souhaiter un ralentissement de la croissance urbaine afin de favoriser le progrès des campagnes. "Ce qui convient, à l'heure présente, c'est que les campagnes se peuplent et que les villes restent stationnaires." 68 Cette affirmation s'explique dans le contexte de ralentissement économique et de chômage qui sévit encore en 1921. ${ }^{69}$ Elle est cependant la preuve d'une grande naïveté chez Pelletier. Le journaliste ne semble même pas se douter que son projet peut entraîner le pays vers une stagnation encore plus grande. Prétendre qu'il faut arrêter le développement des villes

\footnotetext{
${ }^{64}$ Joseph Versailles in Le problème industriel au Canada français (Montréal, ACJC, 1922), 100-111.

65 Le Devoir, 27 juin 1918, 1.

66 Ibid., 25 septembre 1916, 1 .

67 Ibid., 21 octobre 1922, 1.

68 Ibid., 17 octobre 1921, 1.

69 Ibid., 7 octobre 1921, 1.
} 
parce que c'est au tour des campagnes de progresser, c'est mal évaluer les forces véritablement dynamiques dans une économie moderne.

Pelletier prêche surtout le retour à la terre lorsqu'une période de crise économique ralentit la production industrielle. C'est le cas en 1914, au tout début de la guerre. ${ }^{70}$ De même, en 1920, il prône le retour à la terre car le cultivateur est moins exposé que l'ouvrier aux fluctuations économiques. ${ }^{71}$

Le journaliste du Devoir est favorable à une industrialisation modérée, mais il craint les excès d'une trop grande industrialisation. Il juge excessive la concentration des activités économiques dans quelques grandes villes. ${ }^{72}$ Il souhaite que s'établisse entre les villes et les campagnes un équilibre qui laisserait aux régions rurales plus de la moitié de la population.

Tout le monde, cela va de soi, ne peut s'en aller sur la terre, et personne n'entretiendra, s'il a son bon sens, l'idée d'une société exclusivement formée de ruraux. Les villes ont leur raison d'être, à la condition de ne pas englober la moitié ou les trois quarts des habitants d'un pays, ce qui romprait l'équilibre. ${ }^{33}$

Ainsi Pelletier perçoit d'abord l'agriculture comme un mode de vie auquel est attaché un système de valeur, mais aussi comme une activité économique. En effet, il est assez lucide pour constater que la situation concrète de l'agriculture québecoise n'est pas toujours alléchante. Le salaire de l'ouvrier est souvent plus attirant que le revenu du cultivateur. Il se rend bien compte qu'il faut moderniser l'agriculture pour la rendre plus efficace et plus rentable. Il propose lui-même quelques mesures en vue d'améliorer la situation. Le premier moyen consisterait à accentuer l'éducation agricole. ${ }^{74}$ Le journaliste est également sensible au problème du crédit agricole et, selon lui, le succès du retour à la terre est lié au développement de coopératives. ${ }^{75}$ Il suggère à quelques reprises d'augmenter le revenu du cultivateur tout en réduisant celui des intermédiaires, de sorte que le consommateur n'ait pas à débourser plus. En 1927, il demande en outre au gouvernement provincial d'établir un crédit pour le colon. ${ }^{76}$ Il

\footnotetext{
70 Ibid., 25 août $1914,1$.

71 Ibid., 6 décembre 1920, 1.

72 Ibid., 21 novembre 1924, 1.

73 Ibid., 21 octobre 1922, 1.

74 Ibid., 22 décembre 1913, 2.

75 Ibid., 27 août 1914, 1.

76 Ibid., 24 octobre 1927, 1.
} 
réclame l'amélioration des chemins pour mieux commercialiser les produits, la création de "bureaux de placement agricole" dans les villes. ${ }^{77} \mathrm{Il}$ appuie l'idée de créer de petites industries rurales pour occuper les agriculteurs pendant l'hiver. ${ }^{78}$

Les demandes de réforme de Pelletier ont pour but d'améliorer le sort de l'agriculteur et de moderniser l'agriculture. Cependant, en voulant diffuser l'agriculture, Pelletier, comme bien des gens de son époque, semble inconscient qu'une bonne partie des terres arables ne sont guère productives. Il exige de forts investissements pour la colonisation, mais ne montre guère leur rentabilité sur le plan économique. Enfin, il faut, selon lui, établir un lien étroit entre l'industrie et l'agriculture et faire de celle-ci la base de l'industrialisation du Québec.

Les idées de Georges Pelletier sur l'industrie ont déjà été étudiées. ${ }^{79}$ Elles sont exprimées surtout dans deux articles à L'Action française en 1921 et $1922 .{ }^{80}$ Pelletier essaie d'évaluer les causes de l'absence des Canadiens français à la tête des grandes industries. Il mentionne l'inaptitude des gouvernants à prévoir l'industrialisation rapide et l'attitude timorée d'industriels canadiens-français qui n'ont pas su donner à leurs entreprises une ampleur nouvelle. Il énumère les éléments nécessaires à la réussite industrielle :

Une race qui veut occuper dans l'industrie un rang important a besoin, entre autres choses, de matières premières facilement accessibles, d'énergie motrice peu chère, de capitaux assez forts, de chefs et de main-d'œuvre habiles et, finalement de marchés domestiques et extérieurs. ${ }^{81}$

Pelletier observe que les matières premières ont été, en grande partie, concédées à des étrangers; il voit cependant un espoir du côté des ressources situées dans des régions inaccessibles et il demande qu'on les conserve pour les Canadiens

77 Ibid., 22 décembre 1913, 1.

78 Ibid., 8 août $1923,1$.

79 Paul-André Linteau, "Georges Pelletier et la vie économique des Canadiens français", R. Comeau, édit., Economie québécoise (Montréal, Presses de l'Université du Québec, 1969), 405-416.

80 Georges Pelletier, "Notre industrie", L'Action française, V, 6 (juin 1921) : 322-356. A l'avenir, "Notre industrie". Id., "Les obstacles économiques à l'indépendance du Canada français", L'Action française, VIII, 2 (août 1922): 66-82. Ce texte a été repris dans Notre avenir politique (Montréal, 1923), 141-159; nous utilisons cette dernière pagination. A l'avenir, "Les obstacles".

81 "Notre industrie", 338-339. 
français. ${ }^{82}$ Constatant que les champs appartiennent encore aux Canadiens français il propose d'en faire la base de leur industrialisation, d'édifier des entreprises liées de près à l'agriculture (cuir, lainages, beurre, etc.) ${ }^{83} \mathrm{Il}$ en arrive donc à une vision assez tronquée de l'industrialisation en plaçant au premier rang l'industrie agricole. Le journaliste regrette également que l'énergie motrice soit déjà aux mains de l'étranger. Il propose une meilleure utilisation des capitaux canadiens-français, conseille de déposer dans les banques canadiennes-françaises et d'éviter les placements hasardeux. ${ }^{84}$ Ce plan d'action est assez loin du goût du risque et de l'esprit d'entreprise qui caractérisent en général l'industrialisation. La présence massive des capitaux étrangers inquiète Pelletier. Mais il n'est pas du tout question de déposséder ces étrangers même si le Canada français réalise son indépendance. D'ailieurs, ils ont eux-mêmes intérêt à rester ici. $^{85}$

Pour assurer le succès des industries, Pelletier insiste également sur la nécessité de former des chefs ayant une solide instruction et il loue la qualité de la main-d'œuvre canadiennefrançaise. ${ }^{86}$ Quant aux marchés, le journaliste en a une perception assez modeste. Il lui semble utopique de penser à obtenir une place importante sur les marchés étrangers. Il conseille donc aux industriels canadiens-français de concentrer leurs efforts sur le marché intérieur; c'est là qu'ils ont le plus de chances de réussir. ${ }^{87}$

Pelletier a donc une vision assez restreinte de l'industrialisation du Québec: d'envergure moyenne, axée sur l'agriculture, orientée surtout vers le marché québecois. Plus qu'un projet d'industrialisation, Pelletier propose un plan d'autarcie économique dont le moteur serait l'agriculture (à la fois comme mode de vie et comme activité économique) à laquelle les autres agents de la vie économique seraient étroitement liés. La réalisation du plan de Pelletier repose essentiellement sur la bonne volonté. Négligeant de faire appel à un catalyseur d'énergie comme pourrait l'être l'Etat québecois, Pelletier semble condamné à rêver d'indépendance économique sans jamais pouvoir se donner

82 "Les obstacles", 143-144.

83 "Notre industrie", 341-342.

84 Ibid., 343-345; "Les obstacles", 149-150.

85 "Les obstacles", 156-159.

86 "Notre industrie", 346-448.

87 Ibid., 354-355. 
les moyens de la réaliser. Il ne faut d'ailleurs pas se surprendre de cette faiblesse de la pensée du journaliste. Il est tributaire de son milieu et de son époque.

L'élite nationaliste traditionnelle a souvent essuyé le reproche d'avoir négligé les questions économiques. On ne saurait adresser un tel blâme à Georges Pelletier. En effet, celui-ci a manifesté, face aux problèmes économiques, un vif intérêt qui surprend chez un journaliste politique. Il s'est attaqué à des questions ardues, comme celles du coût de la vie ou de l'émancipation économique des Canadiens français. Bien sûr, son analyse économique présente, comme nous l'avons vu, de sérieuses faiblesses. Il ne faut pas s'en surprendre. Pelletier n'est pas un économiste et n'a pas étudié cette discipline. Il s'est formé lui-même au hasard des lectures et des discussions faisant d'ailleurs preuve d'un excellent esprit de recherche et d'un souci de se documenter. Mais au début du siècle, le champ des sciences sociales est encore peuplé d'un grand nombre d'amateurs et les "experts" qu'a pu consulter Pelletier n'étaient probablement pas tous très compétents. Les faiblesses que nous avons soulignées ne sont pas propres à Pelletier; elles sont caractéristiques du milieu et de l'époque. La pensée économique, non seulement au Québec mais au Canada, est encore à l'état embryonnaire à ce moment et bien mince est l'influence des quelques économistes qui œuvrent dans les universités.

La pensée économique de Pelletier est marquée par un certain désir de se suffire à soi-même qu'on pourrait qualifier - le mot est peut-être un peu fort - d'autarcie. Pelletier semble n'avoir pas bien compris la valeur de la division des tâches et de la spécialisation des individus aussi bien que des groupes ou des régions. On trouve aussi chez Pelletier une notion, assez mal définie, d'équilibre: entre le producteur et le consommateur, entre l'industrie et l'agriculture. Le journaliste accepte avec réticence les phénomènes d'urbanisation et d'industrialisation. Il n'a surtout pas saisi dans toute son ampleur la permanence de ces phénomènes. Cependant, il ne s'oppose pas carrément à l'industrialisation. Il veut plutôt la contrôler, la subordonner à l'agriculture dans le but de maintenir un équilibre idéal.

Les propositions de Pelletier, en matière économique, sont marquées par la modération et même le conservatisme. Sa doctrine manque de ce dynamisme conquérant qui fait les succès industriels. Pelletier se signale cependant par sa vigoureuse 
défense des faibles, des petits salariés. Il intervient maintes fois en faveur du consommateur. Il appuie les coopératives, les ligues de consommateurs. Il lutte contre les trusts qui exercent un contrôle des prix. Il réclame une action gouvernementale qui se limitera cependant au contrôle et à la réglementation, afin d'éliminer les abus. Là aussi, Pelletier est bien de son époque. Ses demandes sont parallèles à celles des mouvements agraires dans l'Ouest, des progressistes aux Etats-Unis, bien que ses motifs puissent être différents des leurs.

Malgré son vif intérêt pour les questions économiques et son désir de bien se renseigner, l'analyse de Pelletier présente donc de sérieuses faiblesses. L'étude de sa pensée nous permet néanmoins de mieux saisir les postulats et les objectifs de l'élite traditionnelle du Québec.

Département d'histoire

PAUL-ANDRÉ LINTEAU

Université du Québec

Constituante de Montréal 